\title{
On relaxed and contraction-proximal point algorithms in hilbert spaces
}

\author{
Shuyu Wang ${ }^{*}$ and Fenghui Wang
}

* Correspondence: shyuwang@163. com

Department Of Mathematics, Luoyang Normal University, Luoyang 471022, China

\begin{abstract}
We consider the relaxed and contraction-proximal point algorithms in Hilbert spaces. Some conditions on the parameters for guaranteeing the convergence of the algorithm are relaxed or removed. As a result, we extend some recent results of Ceng-Wu-Yao and Noor-Yao.
\end{abstract}

Keywords: maximal monotone operator, proximal point algorithm, firmly nonexpansive operator

\section{Introduction}

Throughout, $H$ denotes a real Hilbert space and $A$ a multi-valued operator with domain $D(A)$. We know that $A$ is called monotone if $\langle u-v, x-y\rangle \geq 0$, for any $u \in A x$, $v \in A y$; maximal monotone if its graph $G(A)=\{(x, y): x \in D(A), y \in A x\}$ is not properly contained in the graph of any other monotone operator. Denote by $S:=\{x \in D$ $(A): 0 \in A x\}$ the zero set and by $J_{c}:=(I+c A)^{-1}$ the resolvent of $A$. It is well known that $J_{c}$ is single valued and $D\left(J_{c}\right)=H$ for any $c>0$.

A fundamental problem of monotone operators is that of finding an element $x$ so that $0 \in A x$. This problem is essential because it includes many concrete examples, such as convex programming and monotone variational inequalities. A successful and powerful algorithm for solving this problem is the well-known proximal point algorithm (PPA), which generates, for any initial guess, $x_{0} \in H$, an iterative sequence as

$$
x_{n+1}=J_{c_{n}}\left(x_{n}+e_{n}\right) \text {, }
$$

where $\left(c_{n}\right)$ is a positive real sequence and $\left(e_{n}\right)$ is the error sequence (see [1]). To guarantee the convergence of PPA, there are two kinds of accuracy criterion posed on the error sequence:

$$
\begin{aligned}
& \text { (I) }\left\|e_{n}\right\| \leq \varepsilon_{n}, \quad \sum_{n=0}^{\infty} \varepsilon_{n}<\infty \text { or } \\
& \text { (II) }\left\|e_{n}\right\| \leq \eta_{n}\left\|\tilde{x}_{n}-x_{n}\right\|, \quad \sum_{n=0}^{\infty} \eta_{n}<\infty,
\end{aligned}
$$

where $\tilde{x}_{n}=J_{c_{n}}\left(x_{n}+e_{n}\right)$. In 2001, Han and He [2] proved that in finite dimensional Hilbert space criterion (II) can be replaced by 
$\left(\mathrm{II}^{\prime}\right)\left\|e_{n}\right\| \leq \eta_{n}\left\|\tilde{x}_{n}-x_{n}\right\|, \quad \sum_{n=0}^{\infty} \eta_{n}^{2}<\infty$

The infinite version was obtained by Marino and $\mathrm{Xu}$ [3].

There are various generations or modifications on the PPA. Among them Eckstein and Bertsekas [4] proposed the relaxed proximal point algorithm (RPPA):

$$
x_{n+1}=\left(1-\rho_{n}\right) x_{n}+\rho_{n} J_{\mathcal{C}_{n}}\left(x_{n}\right)+e_{n},
$$

where $\left(\rho_{n}\right) \subset(0,2)$ is a relaxation factor. The weak convergence of $(1.2)$ is guaranteed provided that $\left(e_{n}\right)$ satisfies criterion $(\mathrm{I})$,

$$
c_{n} \geq \bar{c}>0, \quad 0<\delta \leq \rho_{n} \leq 2-\delta .
$$

On the other hand, since the PPA does not necessarily converge strongly (see [5]), many authors have conducted worthwhile studies on modifying the PPA so that the strong convergence is guaranteed (see, for instance, [6-8]). In particular, Marino and $\mathrm{Xu}$ [3] proposed the contraction-proximal point algorithm (CPPA):

$$
x_{n+1}=\lambda_{n} u+\left(1-\lambda_{n}\right) J_{c_{n}}\left(x_{n}\right)+e_{n},
$$

where the parameters above satisfy (i) $\lim _{n} \lambda_{n}=0, \Sigma_{n} \lambda_{n}=\infty$; (ii) either $\Sigma_{n}\left|\lambda_{n}+1-\lambda_{n}\right|<\infty$; or $\lim _{n} \lambda_{n} / \lambda_{n}+1=1$; (iii) $0<\underline{c} \leq c_{n} \leq \bar{c}<\infty, \sum_{n}\left|c_{n+1}-c_{n}\right|<\infty$; (iv) $\Sigma_{n}|| e_{n}||<\infty$. Under these assumptions, the CPPA converges strongly to $P_{S}(u)$, the projection of $u$ onto $S$.

In this article, we shall focus on the RPPA and CPPA. We note that the resolvent is in fact the arithmetic mean of the identity and a nonexpansive operator. By using this fact, we relax or remove some sufficient conditions to guarantee the convergence of the algorithms. As a result, we extend and improve some recent results on the PPA.

\section{Some lemmas}

We know that an operator $T: H \rightarrow H$ is called (i) nonexpansive if $\|T x-T y\| \leq \| x-$ $y \| \forall x, y \in H$; and (ii) firmly nonexpansive if $\langle T x-T y, x-y\rangle \geq\|T x-T y\|^{2} \forall x, y \in H$. Denote by $\operatorname{Fix}(T)=\{x \in H: x=T x\}$ the fixed point set of $T$. It is well known that firmly nonexpansive operators have the following properties.

Lemma 1 (Goebel-Kirk [9]). Let $T$ be firmly nonexpansive. Then (1) $2 T$ - I is nonexpansive; (2) $\langle T x-x, T x-z\rangle \leq 0$ for all $x L H$ and for all $z L H \operatorname{Fix}(T)$.

It is well known that $J_{c}$ is firmly nonexpansive and consequently nonexpansive; moreover, $S=$ Fix $\left(J_{c}\right)$. Since the fixed point set of nonexpansive operators is closed convex, the projection $P_{S}$ onto the solution set $S$ is well defined whenever $\mathrm{S} \neq \varnothing$. Hereafter, we assume that $S$ is nonempty. The following lemmas play an important role in our convergence analysis.

Lemma 2 (resolvent identity [3]). Let $c, t>0$. Then for any $x L H$,

$$
J_{c} x=J_{t}\left(\frac{t}{c} x+\left(1-\frac{t}{c}\right) J_{c} x\right) .
$$

Lemma 3 ([10]). Let $\left(\rho_{n}\right)$ be real sequence satisfying

$$
0<\liminf _{n \rightarrow \infty} \rho_{n} \leq \limsup _{n \rightarrow \infty} \rho_{n}<1 .
$$


Assume that $\left(x_{n}\right)$ and $\left(y_{n}\right)$ are bounded sequences in $H$ satisfying $x_{n}+1=\left(1-\rho_{n}\right) x_{n}+$ $\rho_{n} y_{n}$. If

$$
\limsup _{n \rightarrow \infty}\left(\left\|y_{n+1}-y_{n}\right\|-\left\|x_{n+1}-x_{n}\right\|\right) \leq 0,
$$

then $\lim _{n} \rightarrow \infty|| x_{n}-y_{n}||=0$.

Lemma 4 For $r, s,>0$, let $T_{r}=2 J_{r}-I$. Then for any $x \in H$,

$$
\left\|T_{s} x-T_{r} x\right\| \leq\left|1-\frac{s}{r}\right|\left\|x-T_{r} x\right\| .
$$

Proof. Using the resolvent identity, we have

$$
\begin{aligned}
\left\|T_{s} x-T_{r} x\right\| & =2\left\|J_{s} x-J_{s}\left(\frac{s}{r} x+\left(1-\frac{s}{r}\right) J_{r} x\right)\right\| \\
& \leq 2\left\|x-\left(\frac{s}{r} x+\left(1-\frac{s}{r}\right) J_{r} x\right)\right\| \\
& =2\left|1-\frac{s}{r}\right|\left\|x-J_{r} x\right\| \\
& =\left|1-\frac{s}{r}\right|\left\|x-T_{r} x\right\|,
\end{aligned}
$$

where the inequality uses the nonexpansive property of the resolvent.

Lemma 5 ([11]). Let $\left(\varepsilon_{n}\right)$ and $\left(s_{n}\right)$ be positive real sequences. Assume that $\Sigma_{n} \varepsilon_{n}<\infty$. If either (i) $s_{n+} 1 \leq\left(1+\varepsilon_{n}\right) s_{n}$ or (ii) $s_{n+} 1 \leq \varepsilon_{n}$, then the limit of $\left(s_{n}\right)$ exists.

\section{The relaxed proximal point algorithm}

Under criterion (II'), Ceng et al. [12] considered another type, RPPA:

$$
\left\{\begin{array}{l}
\tilde{x}_{n}=J_{c_{n}}\left(x_{n}+e_{n}\right) \\
x_{n+1}=\left(1-\rho_{n}\right) x_{n}+\rho_{n} \tilde{x}_{n}
\end{array}\right.
$$

and proved the weak convergence of (3.1) under the assumptions:

$$
c_{n} \geq \bar{c}>0, \quad 0<\delta \leq \rho_{n} \leq 1 .
$$

We note that the choice of $\left(\rho_{n}\right)$ excludes the case whenever $\rho_{n} \in(1,2)$, the overrelaxation. The overrelaxation, however, may indeed speed up the convergence of the algorithm (see [13]). Below, we shall improve their conditions on the relaxation factor from $0<\delta \leq \rho_{n} \leq 1$ to $0<\delta \leq \rho_{n} \leq 2-\delta$.

Theorem 6. Assume that the following conditions hold:
(a) $c_{n} \geq \bar{c}>0$;
(b) $0<\delta \leq \rho_{n} \leq 2-\delta$;
(c) $\sum_{n}\left\|e_{n}\right\| \leq \eta_{n}\left\|\tilde{x}_{n}-x_{n}\right\|, \sum_{n} \eta_{n}^{2}<\infty$.

Then the sequence generated by (3.1) converges weakly to a point in $S$.

Proof. The key point of our proof is to show $\lim _{n} s_{n}=0$, where $s_{n}=\left\|x_{n}-J_{c_{n}}\left(x_{n}\right)\right\|$. To see this, let $z \in S$ be fixed. Since $J_{c_{n}}$ is firmly nonexpansive and $z \in \operatorname{Fix}\left(J_{c_{n}}\right)$, applying Lemma 1 yields $\left\langle\tilde{x}_{n}-z, \tilde{x}_{n}-x_{n}-e_{n}\right\rangle \leq 0$. This together with (3.1) enables us to get

$$
\begin{aligned}
\left\|x_{n+1}-z\right\|^{2}-\left\|x_{n}-z\right\|^{2} & =\left\|\left(x_{n}-z\right)+\rho_{n}\left(\tilde{x}_{n}-x_{n}\right)\right\|^{2}-\left\|x_{n}-z\right\|^{2} \\
& =2 \rho_{n}\left\langle x_{n}-z, \tilde{x}_{n}-x_{n}\right\rangle+\rho_{n}^{2}\left\|\tilde{x}_{n}-x_{n}\right\|^{2} \\
& =2 \rho_{n}\left\langle\tilde{x}_{n}-z, \tilde{x}_{n}-x_{n}\right\rangle-\rho_{n}\left(2-\rho_{n}\right)\left\|\tilde{x}_{n}-x_{n}\right\|^{2} \\
& \leq 2 \rho_{n}\left(\tilde{x}_{n}-z, e_{n}\right\rangle-\rho_{n}\left(2-\rho_{n}\right)\left\|\tilde{x}_{n}-x_{n}\right\|^{2} \\
& =2 \rho_{n}\left\langle\tilde{x}_{n}-x_{n}, e_{n}\right\rangle+2 \rho_{n}\left\langle x_{n}-z, e_{n}\right\rangle-\rho_{n}\left(2-\rho_{n}\right)\left\|\tilde{x}_{n}-x_{n}\right\|^{2} \\
& \leq 2 \rho_{n}\left\|e_{n}\right\|\left\|\tilde{x}_{n}-x_{n}\right\|+2 \rho_{n}\left\|e_{n}\right\|\left\|x_{n}-z\right\|-\rho_{n}\left(2-\rho_{n}\right)\left\|\tilde{x}_{n}-x_{n}\right\|^{2} \\
& \leq 2 \rho_{n} \eta_{n}\left\|\tilde{x}_{n}-x_{n}\right\|^{2}+2 \rho_{n} \eta_{n}\left\|\tilde{x}_{n}-x_{n}\right\|\left\|x_{n}-z\right\| \\
& -\rho_{n}\left(2-\rho_{n}\right)\left\|\tilde{x}_{n}-x_{n}\right\|^{2} .
\end{aligned}
$$


Using the basic inequality $2 a b \leq a^{2} / \varepsilon+\varepsilon b^{2}(a, b \in \mathbb{R}, \varepsilon>0)$, we arrive at

$$
\begin{aligned}
2 \rho_{n} \eta_{n}\left\|x_{n}-z\right\|\left\|\tilde{x}_{n}-x_{n}\right\| & \leq \frac{2 \rho_{n}}{2-\rho_{n}}\left(\eta_{n}\left\|x_{n}-z\right\|^{2}+\frac{2-\rho_{n}}{2 \rho_{n}}\left(\rho_{n}\left\|\tilde{x}_{n}-x_{n}\right\|\right)^{2}\right. \\
& =\frac{2 \rho_{n} \eta_{n}^{2}}{2-\rho_{n}}\left\|x_{n}-z\right\|^{2}+\frac{\rho_{n}\left(2-\rho_{n}\right)}{2}\left\|\tilde{x}_{n}-x_{n}\right\|^{2} \\
& \leq \frac{2(2-\delta) \eta_{n}^{2}}{\delta}\left\|x_{n}-z\right\|^{2}+\frac{\rho_{n}\left(2-\rho_{n}\right)}{2}\left\|\tilde{x}_{n}-x_{n}\right\|^{2} \\
& =\varepsilon_{n}\left\|x_{n}-z\right\|^{2}+\frac{\rho_{n}\left(2-\rho_{n}\right)}{2}\left\|\tilde{x}_{n}-x_{n}\right\|^{2},
\end{aligned}
$$

where $\varepsilon_{n}=2(2-\delta) \eta_{n}^{2} / \delta$ is a summable sequence. Substituting this into above yields

$$
\left\|x_{n+1}-z\right\|^{2} \leq\left(1+\varepsilon_{n}\right)\left\|x_{n}-z\right\|^{2}-\frac{\rho_{n}\left(2-\rho_{n}-4 \eta_{n}\right)}{2}\left\|\tilde{x}_{n}-x_{n}\right\|^{2} .
$$

Since by Lemma 5 the limit of $\left\|x_{n}-z\right\|^{2}$ exists and $\lim _{\inf } \rho_{n}\left(2-\rho_{n}-4 \eta_{n}\right) \geq \delta(2-\delta)$, this implies that $\left\|\tilde{x}_{n}-x_{n}\right\| \rightarrow 0$. On the other hand, we note that for all $\mathrm{n} \in \mathbb{N}$

$$
s_{n} \leq\left(1+\eta_{n}\right)\left\|x_{n}-\tilde{x}_{n}\right\| \rightarrow 0 ;
$$

therefore, $\lim _{n} s_{n}=0$. The rest proof is similar to that of [12, Theorem 3.1].

We now turn to the RPPA (1.2). Under the criterion (I), the assumptions on relaxation factors can be relaxed to $\Sigma \rho_{n}\left(2-\rho_{n}\right)=\infty$ (see [3, Theorem 3.3]). Since the proof there is very technical, we wang to restate this result with a simple proof.

Theorem 7. Assume that the following conditions hold:

(a) $\Sigma_{n}\left\|e_{n}\right\|<\infty$;

(b) $\Sigma_{n} \rho_{n}\left(2-\rho_{n}\right)=\infty$;

(c) $0<\bar{c} \leq c_{n} \leq \tilde{c}<\infty$;

(d) $\Sigma_{n}\left|c_{n}+1-c_{n}\right|<\infty$.

Then the sequence generated by (1.2) converges weakly to a point in $S$.

Proof. The key step is to show $\lim _{n} s_{n}=0$, where $s_{n}=\left\|x_{n}-J_{c_{n}}\left(x_{n}\right)\right\|$. It has been shown that $\Sigma_{n} \rho_{n}\left(2-\rho_{n}\right) s_{n}<\infty$ (see [3, Lemma 3.2]). Therefore, it remains to show that $\lim _{n} s_{n}$ exists. By letting $T_{n}=2 J_{n}-I$, we rewrite (2) as

$$
x_{n+1}=\left(1-\frac{\rho_{n}}{2}\right) x_{n}+\frac{\rho_{n}}{2} T_{n} x_{n}+e_{n} .
$$

In view of Lemma 4 and condition (c),

$$
\begin{aligned}
\left\|T_{n+1} x_{n+1}-T_{n} x_{n}\right\| & \leq\left\|T_{n+1} x_{n+1}-T_{n+1} x_{n}\right\|+\left\|T_{n+1} x_{n}-T_{n} x_{n}\right\| \\
& \leq\left\|x_{n}-x_{n+1}\right\|+\left\|T_{n+1} x_{n}-T_{n} x_{n}\right\| \\
& \leq\left\|x_{n}-x_{n+1}\right\|+\left|1-\frac{c_{n+1}}{c_{n}}\right|\left\|T_{n} x_{n}-x_{n}\right\| \\
& \leq\left\|x_{n}-x_{n+1}\right\|+\frac{\left|c_{n+1}-c_{n}\right|}{\bar{c}}\left\|T_{n} x_{n}-x_{n}\right\| \\
& \leq\left\|x_{n}-x_{n+1}\right\|+M\left|c_{n+1}-c_{n}\right|,
\end{aligned}
$$


where $M>0$ is a suitable number. Consequently,

$$
\begin{aligned}
\left\|x_{n+1}-T_{n+1} x_{n+1}\right\|= & \left\|\left(1-\frac{\rho_{n}}{2}\right) x_{n}+\frac{\rho_{n}}{2} T_{n} x_{n}+e_{n}-T_{n+1} x_{n+1}\right\| \\
= & \left\|\left(1-\frac{\rho_{n}}{2}\right)\left(x_{n}-T_{n} x_{n}\right)+\left(T_{n} x_{n}-T_{n+1} x_{n+1}\right)+e_{n}\right\| \\
\leq & \left(1-\frac{\rho_{n}}{2}\right)\left\|x_{n}-T_{n} x_{n}\right\|+\left\|T_{n} x_{n}-T_{n+1} x_{n+1}\right\|+\left\|e_{n}\right\| \\
\leq & \left(1-\frac{\rho_{n}}{2}\right)\left\|x_{n}-T_{n} x_{n}\right\|+\left\|x_{n}-x_{n+1}\right\| \\
& +M\left|c_{n+1}-c_{n}\right|+\left\|e_{n}\right\| \\
= & \left(1-\frac{\rho_{n}}{2}\right)\left\|x_{n}-T_{n} x_{n}\right\|+\left\|\frac{\rho_{n}}{2}\left(x_{n}-T_{n} x_{n}\right)+e_{n}\right\| \\
& +M\left|c_{n+1}-c_{n}\right|+\left\|e_{n}\right\| \\
\leq & \left\|x_{n}-T_{n} x_{n}\right\|+M\left|c_{n+1}-c_{n}\right|+2\left\|e_{n}\right\| .
\end{aligned}
$$

Using $s_{n}=\left\|x_{n}-T_{n} x_{n}\right\| / 2$, we therefore arrive at

$$
s_{n+1} \leq s_{n}+\sigma_{n}
$$

where $\sigma_{n}=2 M\left|c_{n}+1-c_{n}\right|+4|| e_{n}||$ satisfying $\Sigma_{n} \sigma_{n}<\infty$ (due to (a) and (d)). By Lemma 5, we finally conclude that $\lim _{n} s_{n}=0$.

\section{The contraction-proximal point algorithm}

Recently, Yao and Noor [14] extended the CPPA to the following form:

$$
x_{n+1}=\lambda_{n} u+r_{n} x_{n}+\delta_{n} J_{c_{n}}\left(x_{n}\right)+e_{n}
$$

where $\left(\lambda_{n}\right),\left(r_{n}\right),\left(\delta_{n}\right) \subseteq(0,1)$ and $\lambda_{n}+r_{n}+\delta_{n}=1$. They proved the strong convergence of the algorithm provided that (i) $c_{n} \geq \bar{c}>0, \lim _{n}\left|c_{n+1}-c_{n}\right|=0$; (ii) $0<\lim \inf _{n} r_{n} \leq$ $\lim \sup _{n} r_{n}<1$; and (iii) $\Sigma_{n}\left\|e_{n}\right\|<\infty$. Also, they claimed that their algorithm includes the CPPA as a special case. This is, however, not the case, because condition (ii) excludes the special case $r_{n} \equiv 0$. To overcome this drawback, we shall show the same result by replacing condition (ii) with the weak condition:

$$
\limsup _{n \rightarrow \infty} r_{n}<1 \Leftrightarrow \liminf _{n \rightarrow \infty} \delta_{n}>0 .
$$

In this situation, the CPPA is evidently a special case of algorithm (4.1). The idea of the following proof is followed by the second author [15].

Theorem 8. Let be $\left(\lambda_{n}\right),\left(r_{n}\right)$ and $\left(\delta_{n}\right)$ be parameters in (4.1). Assume that the following conditions hold:

(a) $\lim _{n} \lambda_{n}=0, \Sigma_{n} \lambda_{n}=\infty$;

(b) $\lim \sup _{n} r_{n}<1 \Leftrightarrow \lim \inf _{n} \delta_{n}>0$;

(c) $c_{n} \geq \bar{c}>0,\left|c_{n+1}-c_{n}\right| \rightarrow 0$;

(d) $\Sigma_{n}|| e_{n} \|<\infty$.

Then the sequence generated by (4.1) converges strongly to $P_{S}(u)$.

Proof. All we need to do is to prove $\left\|x_{n}+1-x_{n}\right\| \rightarrow 0$, since the rest proof is similar to that of [14, Theorem 3.3]. To this end, set $J_{n}=J_{\mathcal{C}_{n}}$ and $T_{n}=2 J_{n}-I$. It then follows from (4.1) that

$$
\begin{aligned}
x_{n+1} & =\lambda_{n} u+r_{n} x_{n}+\frac{\delta_{n}}{2}\left(I+T_{n}\right) x_{n}+e_{n} \\
& =\left(r_{n}+\frac{\delta_{n}}{2}\right) x_{n}+\lambda_{n} u+\frac{\delta_{n}}{2} T_{n} x_{n}+e_{n} .
\end{aligned}
$$


Let $\rho_{n}=\lambda_{n}+\left(\delta_{n} / 2\right)$. Then the algorithm has the form:

$$
x_{n+1}=\left(1-\rho_{n}\right) x_{n}+\rho_{n} y_{n}
$$

where $y_{n}=\left(2 \lambda_{n} u+\delta_{n} T_{n} x_{n}+2 e_{n}\right) / 2 \rho_{n}$. Using nonexpansiveness of $T_{n}$ and Lemma 4, we have

$$
\begin{aligned}
\left\|T_{n+1} x_{n+1}-T_{n} x_{n}\right\| & \leq\left\|T_{n+1} x_{n+1}-T_{n+1} x_{n}\right\|+\left\|T_{n+1} x_{n}-T_{n} x_{n}\right\| \\
& \leq\left\|x_{n+1}-x_{n}\right\|+\left|1-\frac{c_{n+1}}{c_{n}}\right|\left\|T_{n} x_{n}-x_{n}\right\| \\
& \leq\left\|x_{n+1}-x_{n}\right\|+\frac{\left|c_{n}-c_{n+1}\right|}{\bar{c}}\left\|T_{n} x_{n}-x_{n}\right\| .
\end{aligned}
$$

On the other hand, it follows from the definition of $y_{n}$ that

$$
\begin{aligned}
\left\|y_{n+1}-y_{n}\right\|= & \| \frac{1}{2 \rho_{n+1}}\left(2 \lambda_{n+1} u+\delta_{n+1} T_{n+1} x_{n+1}+2 e_{n+1}\right) \\
& -\frac{1}{2 \rho_{n}}\left(2 \lambda_{n} u+\delta_{n} T_{n} x_{n}+2 e_{n}\right) \| \\
\leq & \left|\frac{\lambda_{n+1}}{\rho_{n+1}}-\frac{\lambda_{n}}{\rho_{n}}\right|\|u\|+\frac{\left\|e_{n+1}\right\|}{\rho_{n+1}}+\frac{\left\|e_{n}\right\|}{\rho_{n}} \\
& +\left\|\frac{\delta_{n+1}}{2 \rho_{n+1}} T_{n+1} x_{n+1}-\frac{\delta_{n}}{2 \rho_{n}} T_{n} x_{n}\right\| \\
\leq & \left|\frac{\lambda_{n+1}}{\rho_{n+1}}-\frac{\lambda_{n}}{\rho_{n}}\right|\|u\|+\frac{\left\|e_{n+1}\right\|}{\rho_{n+1}}+\frac{\left\|e_{n}\right\|}{\rho_{n}} \\
& +\left|\frac{\delta_{n+1}}{2 \rho_{n+1}}-\frac{\delta_{n}}{2 \rho_{n}}\right|\left\|T_{n+1} x_{n+1}\right\| \\
& +\frac{\delta_{n}}{2 \rho_{n}}\left\|T_{n+1} x_{n+1}-T_{n} x_{n}\right\| .
\end{aligned}
$$

Since $\left(x_{n}\right)$ is bounded and $T_{n}$ is nonexpansive, we can find $M>0$ so that $\left(\left\|T_{n} x_{n}\right\|+\right.$ $\left.\left\|x_{n}\right\|+\|u\|\right) \leq M$ for all $n \in \mathbb{N}$ Adding (4.3) and (4.4) and noting $\delta_{n} \leq 2 \rho_{n}$ yield

$$
\begin{aligned}
\left\|y_{n+1}-y_{n}\right\| \leq & \left|\frac{\lambda_{n+1}}{\rho_{n+1}}-\frac{\lambda_{n}}{\rho_{n}}\right|\|u\|+\frac{\left\|e_{n+1}\right\|}{\rho_{n+1}}+\frac{\left\|e_{n}\right\|}{\rho_{n}} \\
& +\left|\frac{\delta_{n+1}}{2 \rho_{n+1}}-\frac{\delta_{n}}{2 \rho_{n}}\right|\left\|T_{n+1} x_{n+1}\right\| \\
& +\left\|x_{n+1}-x_{n}\right\|+\frac{\left|c_{n}-c_{n+1}\right|}{\bar{c}}\left\|T_{n} x_{n}-x_{n}\right\| \\
\leq & \left\|x_{n+1}-x_{n}\right\|+M\left(\left|\frac{\lambda_{n+1}}{\rho_{n+1}}-\frac{\lambda_{n}}{\rho_{n}}\right|+\frac{\left\|e_{n+1}\right\|}{\rho_{n+1}}\right. \\
& \left.+\frac{\left\|e_{n}\right\|}{\rho_{n}}+\left|\frac{\delta_{n+1}}{2 \rho_{n+1}}-\frac{\delta_{n}}{2 \rho_{n}}\right|+\frac{\left|c_{n}-c_{n+1}\right|}{\bar{c}}\right) .
\end{aligned}
$$

With the knowledge that $\left\|e_{n}\right\| \rightarrow 0$ and

$$
\frac{\lambda_{n}}{\rho_{n}}=\frac{2 \lambda_{n}}{2 \lambda_{n}+\delta_{n}} \rightarrow 0, \quad \frac{\delta_{n}}{2 \rho_{n}}=\frac{\delta_{n}}{2 \lambda_{n}+\delta_{n}} \rightarrow 1,
$$


we therefore deduce from (b) and (c) that

$$
\begin{aligned}
& \underset{n \rightarrow \infty}{\limsup }\left(\left\|y_{n+1}-y_{n}\right\|-\left\|x_{n+1}-x_{n}\right\|\right) \\
& \quad \leq \limsup _{n \rightarrow \infty} M\left(\left|\frac{\lambda_{n+1}}{\rho_{n+1}}-\frac{\lambda_{n}}{\rho_{n}}\right|+\frac{\left\|e_{n+1}\right\|}{\rho_{n+1}}+\frac{\left\|e_{n}\right\|}{\rho_{n}}\right. \\
& \left.\quad+\left|\frac{\delta_{n+1}}{2 \rho_{n+1}}-\frac{\delta_{n}}{2 \rho_{n}}\right|+\frac{\left|c_{n}-c_{n+1}\right|}{\bar{c}}\right) \rightarrow 0 .
\end{aligned}
$$

Note that $\lim \inf _{n} \rho_{n}=\lim \inf _{n}\left(\delta_{n} / 2\right)>0$ and $\lim \sup _{n} \rho_{n}=\lim \sup _{n}\left(\delta_{n} / 2\right) \leq 1 / 2<1$. On the other hand, it is easy to check that $\left(x_{n}\right)$ is bounded and so is $\left(y_{n}\right)$ We therefore apply Lemma 3 to yield $\lim _{n}\left\|x_{n}-y_{n}\right\|=0$. By means of (4.2), we finally have

$$
\left\|x_{n+1}-x_{n}\right\|=\rho_{n}\left\|x_{n}-y_{n}\right\| \rightarrow,
$$

and thus the required result at once follows.

As a corollary, we improve [3, Theorem 4.1] as follows.

Theorem 9. Assume that the following conditions hold:

(a) $\lim _{n} \lambda_{n}=0, \Sigma_{n} \lambda_{n}=\infty$;

(b) $c_{n} \geq \bar{c}>0,\left|c_{n+1}-c_{n}\right| \rightarrow 0$;

(c) $\Sigma_{n}\left\|e_{n}\right\|<\infty$

Then the sequence generated by (1.4) converges strongly to $P_{S}(u)$.

\section{Abbreviations}

CPPA: contraction-proximal point algorithm; PPA: proximal point algorithm; RPPA: relaxed proximal point algorithm.

\section{Acknowledgements}

The authors would like to express thier sincere thanks to the referees for their valuable suggestions. This study is supported by the Natural Science Foundation of Department of Education, Henan(2011B110023).

\section{Authors' contributions}

Both authors contributed equally to this work. All authors read and approved the final manuscript.

\section{Competing interests}

The authors declare that they have no competing interests.

Received: 13 March 2011 Accepted: 25 August 2011 Published: 25 August 2011

\section{References}

1. Rockafellar, RT: Monotone operators and the proximal point algorithm. SIAM J Control Optim. 14, $877-898$ (1976). doi:10.1137/0314056

2. Han, D, He, BS: A new accuracy criterion for approximate proximal point algorithms. J Math Anal Appl. 263, 343-354 (2001). doi:10.1006/jmaa.2001.7535

3. Marino, G, Xu, HK: Convergence of generalized proximal point algorithm. Comm Pure Appl Anal. 3, $791-808$ (2004)

4. Eckstein, J, Bertsekas, DP: On the Douglas-Rachford splitting method and the proximal points algorithm for maximal monotone operators. Math Programming. 55, 293-318 (1992). doi:10.1007/BF01581204

5. Güler, O: On the convergence of the proximal point algorithm for convex optimization. SIAM J Control Optim. 29, 403-419 (1991). doi:10.1137/0329022

6. Bauschke, HH, Combettes, PL: A weak-to-strong convergence principle for Fejér-monotone methods in Hilbert spaces. Math Oper Res. 26, 248-264 (2001). doi:10.1287/moor.26.2.248.10558

7. Solodov, MV, Svaiter, BF: Forcing strong convergence of proximal point iterations in a Hilbert space. Math Programming Ser. 87, 189-202 (2000)

8. Xu, HK: Iterative algorithms for nonlinear operators. J Lond Math Soc. 66, 240-256 (2002). doi:10.1112/ S0024610702003332

9. Goebel, K, Kirk, WA: Topics on Metric Fixed Point Theory. Cambridge University Press, Cambridge (1990)

10. Suzuki, T: A sufficient and necessary condition for Halpern-type strong convergence to fixed points of nonexpansive mappings. Proc Am Math Soc. 135, 99-106 (2007)

11. Tan, KK, Xu, HK: Approximating fixed points of nonexpansive mappings by the Ishikawa iteration process. J Math Anal Appl. 178, 301-308 (1993). doi:10.1006/jmaa.1993.1309

12. Ceng, LC, Wu, SY, Yao, JC: New accuracy criteria for modified approximate proximal point algorithms in Hilbert space. Taiwan J Math. 12, 1691-1705 (2008) 
13. Eckstein, J, Ferris, MC: Operator-splitting methods for monotone affine variational inequalities, with a parallel application to optimal control. INFORMS J Comput. 10, 218-235 (1998). doi:10.1287/ijoc.10.2.218

14. Yao, Y, Noor, MA: On convergence criteria of generalized proximal point algorithms. J Comput Appl Math. 217, 46-55 (2008). doi:10.1016/j.cam.2007.06.013

15. Wang, F: A note on the regularized proximal point algorithm. J Global Optim. 50, 531-535 (2011). doi:10.1007/s10898010-9611-z

doi:10.1186/1029-242X-2011-41

Cite this article as: Wang and Wang: On relaxed and contraction-proximal point algorithms in hilbert spaces. Journal of Inequalities and Applications 2011 2011:41.

Submit your manuscript to a SpringerOpen ${ }^{\circ}$ journal and benefit from:

- Convenient online submission

- Rigorous peer review

- Immediate publication on acceptance

- Open access: articles freely available online

- High visibility within the field

- Retaining the copyright to your article

Submit your next manuscript at $\boldsymbol{s p r i n g e r o p e n . c o m ~}$ 